Textures and Microstructures, 1984, Vol. 6, pp. 81-96

0730-3300/84/0602-0081 $\$ 18.50 / 0$

(C) Gordon and Breach Science Publishers Inc. and OPA Ltd., 1984

Printed in Great Britain

\title{
Texture Transformation
}

\author{
H. J. BUNGE†, M. HUMBERT $\ddagger$ and P. I. WELCH† \\ † Institut für Metallkunde und Metallphysik der T. U. Clausthal, F.R.G. \\ $\ddagger$ Laboratoire de Métallurgie Structurale, Université de Metz, France.
}

(Received September 5, 1983)

\section{INTRODUCTION}

Solid state phase transformations, especially martensitic ones, may occur in a crystallographically well-determined way. The best known orientation relationships are the ones due to Kurdjumov-Sachs and Nishiyama- Wassermann which are observed during martensitic phase changes from the $\gamma$ to $\alpha$ phases in steels. If the material, before transformation, has a preferred orientation of its crystallites, i.e. a texture, then it will also have a texture in the transformed $\alpha$ state which is determined by the $\gamma$-texture and the $\gamma \rightarrow \alpha$ orientation relationship. This is called texture transformation.

The orientation relationship between the disappearing and the newly formed crystals is generally not an exact one. The newly formed crystals may deviate more or less from their ideal, crystallographically determined orientation, the probability becoming, however, smaller and smaller as the deviation increases. In the most general case of a solid state transformation, the crystals of the newly formed phase may have a crystal symmetry different from that of the disappearing phase. The crystal symmetry of the disappearing phase gives rise to several symmetrically equivalent variants of the orientation relationships' e.g. 24 in the case of the Kurdjumov-Sachs relation and 12 in the case of the Nishiyama-Wasserman relation. These variants are equivalent with respect to the crystal symmetry of the disappearing phase. Hence, they should occur with equal probability. 
They are, however, not equivalent with respect to the sample coordinate system e.g. rolling direction, transverse direction and normal direction in a sheet. The probabilities of the variants may thus become different. This effect has been called "variant selection". Several possible reasons for variant selection have been discussed. Experimental investigation shows that variant selection depends strongly on the state of the material before transformation.

The problem of texture transformation has been treated quantitatively in terms of the series expansion method taking into account the probability of the deviation from the ideal orientation relations as well as different crystal symmetries in the disappearing and newly formed phases. The problem of variant selection has, however, only been treated qualitatively. In the following, we give a quantitative treatment of variant selection in texture transformation.

\section{THE ORIENTATION DISTRIBUTION FUNCTION}

In order to define the orientation of a crystallite in a polycrystalline sample we fix a coordinate system $K_{A}$ in the sample e.g. rolling direction, transverse direction and normal direction in a sheet. Another coordinate system $K_{B}$ is fixed to the crystal axes e.g. the three cube axes in cubic crystals. The crystal orientation is defined by the rotation $g$ which moves a coordinate system from the position $K_{A}$ to the position $K_{B}$.

$$
K_{B}=g \cdot K_{A}
$$

The rotation $g$ may be described for example by the three Eulerian angles

$$
g=\left\{\varphi_{1} \Phi \varphi_{2}\right\}
$$

The volume fraction of crystals having the orientation $g$ is given by the orientation distribution function (Bunge 1969, 1983).

$$
\frac{d V}{V}=f(g) d g
$$

The texture function $f(g)$ is invariant with respect to certain rotations $g^{c}$ of the crystal coordinate system which form the rotational subgroup of the crystal symmetry group. The function is also invariant with 
respect to certain rotations $g^{s}$ of the sample coordinate system $K_{A}$ which form the rotational subgroup of the sample symmetry.

$$
\dot{\check{f}}\left(g^{c} \cdot g \cdot g^{s}\right)=\dot{\check{f}}(g)
$$

The sample symmetry is symbolized by dots placed above the functional symbol $f$ on the right-hand side, the crystal symmetry by dots on the left side. Different symmetry groups are distinguished by a different number of dots.

\section{THE IDEAL ORIENTATION RELATION}

The disappearing phase may be called the $\gamma$-phase, the newly formed one the $\alpha$-phase. A crystal of the $\gamma$-phase may have the orientation $g_{\gamma}$. It transforms into $\alpha$-crystals, the orientations of which are called $g_{\alpha}^{n}$. They are related to the orientation $g_{\gamma}$ by rotations $\Delta g_{n}$, the $N$ symmetrically equivalent variants of the orientation relation

$$
g_{\alpha}^{n}=\Delta g_{n} \cdot g_{\gamma}
$$

Equation (5) means that the orientation $g_{\alpha}^{n}$ is obtained by carrying out first the rotation $g_{\gamma}$ and then the rotation $\Delta g_{n}$. The orientation distribution function of the $\alpha$-crystals is then obtained from that of the $\gamma$-crystals by the equation

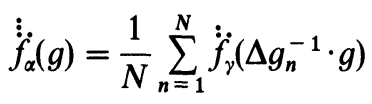

The texture function $f_{\alpha}(g)$ has the same sample symmetry as the function $f_{\gamma}(g)$, but it may have another crystal symmetry.

\section{THE ORIENTATION TRANSFORMATION FUNCTION}

In real cases the orientation relation $\Delta g$ and its symmetrically equivalents $\Delta g_{n}$ may not be the only ones to occur. Rather, there may be a certain probability $w(\Delta g)$ for any orientation relation $\Delta g$ to occur. The function $w(\Delta g)$ will be called the texture transformation function. It was first introduced by Bunge $(1969$, p. 210$)$ in connection with the transformation of textures by recrystallization. It has also been termed the misorientation distribution function (Sargent 1974). 
The rotation $\Delta g$ starts from the crystal coordinate system $K_{B}^{\gamma}$ of the disappearing $\gamma$-crystal and it leads to the crystal coordinate system $K_{B}^{\alpha}$ of the $\alpha$-crystal. The probability function $w(\Delta g)$ must therefore fulfil the symmetries of these crystals as the right and left-hand symmetry respectively (similar to eq. (4)).

The orientation distribution function of the $\alpha$-crystals is obtained from the integral over all orientation relations $\Delta g$ weighted according to the probability of their occurrence (which may be zero for many values $\Delta g$ in the case of a sharp orientation relation (Bunge 1969, 1982, Sargent 1974, Wagner et al. 1981).

$$
\dot{\vdots}_{\alpha}(g)=\int_{\Delta g} \dot{\vdots} \dot{w}(\Delta g) \cdot \dot{\mathscr{f}}_{\gamma}\left(\Delta g^{-1} \cdot g\right) d \Delta g
$$

In special cases the symmetry of the $\alpha$-crystals can of course be the same as the symmetry of the disappearing $\gamma$-crystals. In practice this case is of great importance in the $\gamma \rightarrow \alpha$ transformation in steels.

\section{THE VARIANT SELECTION FUNCTION}

Equation (5) gives the relation between the orientation of the $\gamma$-crystal, the orientation relationship $\Delta g$ and the orientation of the $\alpha$-crystal. The crystal orientation $g_{\gamma}$ is assumed to correspond to the crystal symmetry of the $\gamma$-crystal, i.e. the orientations

$$
g_{\gamma}^{i}=g_{i}^{c} \cdot g_{\gamma}
$$

are equivalent. The function $f(g)$ is invariant with respect to eq. (8) as is shown in eq. (4). Furthermore, the function $w(\Delta g)$ in eq. (7) has been assumed to correspond (on the right-hand side) to the crystal symmetry of the $\gamma$-crystals (on the left side it corresponds to the symmetry of the $\alpha$-crystals). Hence, $\Delta g$ in eq. (7) is equivalent to

$$
\Delta g_{j}=\Delta g \cdot g_{j}^{c}
$$

In fact, if $\Delta g$ is one of the ideal orientation relations $\Delta g_{n}$ in eq. (5), say $\Delta g_{1}$, then the $\Delta g_{j}$ in eq. (9) are identical to the $\Delta g_{n}$. We thus obtain for the orientations of the $\alpha$-crystal

$$
\left\{g_{\alpha}\right\}=\Delta g \cdot g_{j}^{c} \cdot g_{i}^{c} \cdot g_{\gamma}=\Delta g \cdot g_{i}^{c} \cdot g_{\gamma}
$$


In this equation the crystal symmetry elements $g_{i}^{c}$ and $g_{j}^{c}$ run through the whole rotational subgroup of the crystal symmetry. The result is thus the same if the crystal symmetry is taken into account only once. For example, we can omit it in eq. (9) as is shown in the second part of eq. (10). In other words, we can omit the symmetry of the $\gamma$-crystals in the orientation transformation function $w(\Delta g)$ which is then no longer symmetrical on the right-hand side. In terms of an ideal orientation relation, it means that it is sufficient to choose just one of the equivalent orientation relations $\Delta g_{n}$, say $\Delta g_{1}$. In terms of the distribution function $w(\Delta g)$ this function takes on a maximum value at $\Delta g_{1}$ and is zero at all the other $\Delta g_{n}$.

The symmetry of the $\gamma$-crystal is still fully taken into account by the symmetry of the orientation distribution function $f_{y}(g)$. Equation (7) can thus be written in the form

$$
\dot{\vdots} \dot{f}_{\alpha}(g)=\int_{\Delta g} \dot{\vdots}(\Delta g) \cdot \dot{f_{\gamma}}\left(\Delta g^{-1} \cdot g\right) d \Delta g
$$

where $w(\Delta g)$ has now no symmetry on the right-hand side (triclinic symmetry); it has, however, the symmetry of the $\alpha$-crystal on the lefthand side. In eq. (11) the equivalence of the variants of the transformation law with respect to the $\gamma$-symmetry is enforced by eq. (8), which means the invariance of the texture function $f(g)$ over $g^{c}$ according to eq. (4). Equation (11) is thus equivalent to eq. (7), i.e. it describes the texture transformation, still without variant selection.

The invariance of the texture function with crystal symmetry must be valid in any case since the orientations $g_{\gamma}^{i}$ in eq. (8) are indistinguishable crystallographically. They must, however, be distinguished from the transformation point of view i.e. they are considered to be the starting orientations for the variants of the transformation relation which, itself, has now been written without $\gamma$-crystal symmetry. The volume fraction of the newly formed $\alpha$-crystals for the different variants can now be assumed to be proportional to two factors. Firstly, it is proportional to the volume fraction $f_{\gamma}\left(g_{\gamma}\right)$ of the $\gamma$-crystals present, which is the same for all orientation variants, and secondly it is assumed to be proportional to another factor, the variant selection function $v\left(g_{\gamma}\right)$, which also depends on the orientation of the decaying $\gamma$-crystals but which does not obey the $\gamma$-crystal symmetry, i.e. it is different for the different variants. The variant selection function is, however, assumed to have the sample symmetry. Equation (11) can thus be generalized to the case including variant selection. 


$$
\grave{\dot{f}}_{\alpha}(g)=\int_{\Delta g} \dot{\vdots} w(\Delta g) \cdot v\left(\Delta g^{-1} \cdot g\right) \cdot \dot{\dot{f}}_{\gamma}\left(\Delta g^{-1} \cdot g\right) d \Delta g
$$

where $v\left(\Delta g^{-1} \cdot g\right)=v\left(g_{\gamma}\right)$ is the variant selection function. Equation (12) can be rewritten in the form

$$
\grave{\dot{f}_{\alpha}}(g)=\int_{\Delta g} \dot{\dot{w}}(\Delta g) \cdot u\left(\Delta g^{-1} \cdot g\right) d \Delta g
$$

where

$$
\dot{u}\left(g_{\gamma}\right)=\dot{v}\left(g_{\gamma}\right) \cdot \ddot{\dot{f}_{\gamma}}\left(g_{\gamma}\right)
$$

is a function corresponding to the sample symmetry but not to the crystal symmetry of the $\gamma$-crystals.

\section{SERIES EXPANSION}

In order to solve Eq. (13), the functions $f_{\gamma}, f_{\alpha}, v, w$ are developed into series of generalized spherical harmonics of the corresponding symmetries

$$
\begin{gathered}
\dot{f}_{\gamma}(g)=\sum_{l_{1}=0}^{\infty} \sum_{\mu=1}^{M\left(l_{1}\right)} \sum_{v_{1}=1}^{N\left(l_{1}\right)}{ }^{\gamma} C_{l_{1}}^{\mu v_{1}} \dot{\dot{T}}_{l_{1}}^{\mu v_{1}}(g) \\
\dot{f}_{\alpha}(g)=\sum_{l=0}^{\infty} \sum_{\rho=1}^{R(l)} \sum_{v=1}^{N(l)}{ }^{\alpha} C_{l}^{\rho v} \dot{\grave{T}}_{l}^{\rho v}(g) \\
\dot{u}(g)=\sum_{l=0}^{\infty} \sum_{s=-l}^{+l} \sum_{v=1}^{N(l)} U_{l}^{s v} \dot{T}_{l}^{s v}(g) \\
\dot{v}(g)=\sum_{l_{2}=0}^{\infty} \sum_{r=-l_{2}} \sum_{v_{2}=1}^{N\left(l_{2}\right)} V_{l_{2}}^{r v_{2}} \dot{T}_{l_{2}}^{r v_{2}}(g) \\
\dot{\dot{w}}(g)=\sum_{l=0}^{\infty} \sum_{\rho=1}^{R(l)} \sum_{s=-1}^{+l} W_{l}^{\rho s} \dot{\dot{T}}_{l}^{\rho s}(g)
\end{gathered}
$$

It has been shown that the integral relation eq. (13) leads to a relation between the corresponding coefficients (see eq. (10.9) Bunge 1969) 


$$
{ }^{\alpha} C_{l}^{\rho v}=\frac{1}{2 l+1} \sum_{s=-l}^{+l} W_{l}^{\rho s} \cdot U_{l}^{s v}
$$

The function $u$ in eq. (14) is the product of two functions which have the same right-hand side symmetry but different left side symmetries. Therefore we express the left side symmetry of $f_{\gamma}(g)$ by the symmetry coefficients according to the definition of the symmetric functions

$$
\ddot{T}_{l_{1}}^{\mu v_{1}}(g)=\sum_{m=-l_{1}}^{+l_{1}} \dot{A}_{l_{1}}^{m \mu} T_{l_{1}}^{m v_{1}}(g)
$$

We thus obtain

$$
\ddot{\tilde{f}_{\gamma}}(g)=\sum_{l_{1}=0}^{\infty} \sum_{\mu=1}^{M\left(l_{1}\right)} \sum_{v_{1}=1}^{N\left(l_{1}\right)} \sum_{m=-l_{1}}^{+l_{1}}{ }^{\gamma} C_{l_{1}}^{\mu v_{1}} \dot{A}_{l_{1}}^{m \mu} T_{l_{1}}^{m v_{1}}
$$

which we may rewrite

$$
\ddot{f}_{y}(g)=\sum_{l_{1}=0}^{\infty} \sum_{m=-l_{1}}^{+l_{1}} \sum_{v_{1}=1}^{N\left(l_{1}\right)} C_{l_{1}}^{m v_{1}} T_{l_{1}}^{m v_{1}}(g)
$$

with the coefficients

$$
C_{l_{1}}^{m v_{1}}=\sum_{\mu=1}^{M\left(l_{1}\right)}{ }^{\gamma} C_{l_{1}}^{\mu \nu_{1}} \dot{\dot{A}}_{l_{1}}^{m \mu}
$$

The coefficients $C_{l_{1}}^{m v_{1}}$ represent the same texture function $f_{\gamma}(g)$ as the coefficients ${ }^{\gamma} C_{l_{1}}^{\mu v_{1}}$. However, they do not show the crystal symmetry of $\gamma$ explicitely. The function $f_{\gamma}(g)$ is written in eq. (23) formally as a function with triclinic crystal symmetry. With these coefficients, the function $u(g)$ can be written in the form

$$
\dot{u}(g)=\sum_{l_{1}=0}^{\infty} \sum_{m=-l_{1}}^{+l_{1}} \sum_{v_{1}=1}^{N\left(l_{1}\right)} \sum_{l_{2}=0}^{\infty} \sum_{r=-l_{2}}^{+l_{2}} \sum_{v_{2}=1}^{N\left(l_{2}\right)} C_{l_{1}}^{m v_{1}} V_{l_{2}}^{r v_{2}} T_{l_{1}}^{m v_{1}}(g) T_{l_{2}}^{v_{2}}(g)
$$

in which the two functions $T$ have the same right- and left-hand symmetries respectively. The product of two harmonic functions can be expressed by a sum of functions using the Clebsch-Gordan coefficients. 


$$
\dot{T}_{l_{1}}^{m v_{1}}(g) \cdot T_{l_{2}}^{i v_{2}}(g)=\sum_{l=\left|l_{2}-l_{1}\right|}^{\left|l_{2}+l_{1}\right|} \sum_{v=1}^{N(l)}\left(l_{1} l_{2} m r \mid l s\right)\left\{l_{1} l_{2} v_{1} v_{2} \mid l v\right\} T_{l}^{s v}(g)
$$

where $\left(l_{1} l_{2} m r \mid l s\right)$ are the usual Clebsch-Gordan coefficients and $\left\{l_{1} l_{2} v_{1} v_{2} \mid l v\right\}$ are the coefficients for symmetric functions corresponding to the sample symmetry. In eq. (26) $m, r$ and $s$ are related by

$$
s=m+r
$$

The coefficients $\left(l_{1} l_{2} m r \mid l s\right)$ are zero for $s>l$.

The symmetric Clebsch-Gordan coefficients are defined by (Bunge 1968, 1982)

$$
\left\{l_{1} l_{2} v_{1} v_{2} \mid l v\right\}=\sum_{n_{1}=-l_{1}}^{+l_{1}} \sum_{n_{2}}^{+l_{2}}\left(l_{2} l_{1} n_{1} n_{2} \mid l n\right) \dot{A}_{l_{1}}^{n_{1} v_{1}} \dot{A}_{l_{2}}^{n_{2} v_{2}} \dot{A}_{l}^{n v}
$$

The function $u$ eq. (25) can thus be expressed according to eq. (17) with the coefficients

$$
U_{l}^{s v}=\sum_{l_{1}=0}^{\infty} \sum_{l_{2}=0}^{\infty} \sum_{m=-l_{1}}^{+l_{1}} \sum_{v_{1}=1}^{N\left(l_{1}\right)} \sum_{v_{2}=1}^{N\left(l_{2}\right)} C_{l_{1}}^{m v_{1}} V_{l_{2}}^{r v_{2}} \cdot\left(l_{1} l_{2} m r \mid l s\right)\left\{l_{1} l_{2} v_{1} v_{2} \mid l v\right\}
$$

For given values of $l s v$ in eq. (29), eq. (27) is to be taken into account as well as the conditions $s \leqslant l$ (otherwise the coefficients $\left(l_{1} l_{2} m r \mid l s\right)$ are zero). The relationship between the coefficients of the $\alpha$-texture and thøse of the $\gamma$-texture is thus given by combining eq. (20), (29) and (24)

$$
\begin{aligned}
{ }^{\alpha} C_{l}^{\rho v}=\frac{1}{2 l+1} \sum_{s=-l l_{1}=0}^{+l} \sum_{l_{2}=0}^{\infty} \sum_{m=-l_{1}}^{\infty} \sum_{v_{1}=1}^{+l_{1}} \sum_{v_{2}=1}^{N\left(l_{1}\right)} \sum_{\mu=1}^{N\left(l_{2}\right)} \sum_{l}^{M\left(l_{1}\right)} W_{l}^{\rho s} . \\
{ }^{\gamma} C_{l_{1}}^{\mu v_{1}} \cdot V_{l_{2}}^{r v_{2}} \cdot \dot{A}_{l_{1}}^{m \mu}\left(l_{1} l_{2} m r \mid l s\right)\left\{l_{1} l_{2} v_{1} v_{2} \mid l v\right\}
\end{aligned}
$$

With the above mentioned conditions for the indices.

Equation (30) corresponds to eq. (12) expressed in terms of the respective coefficients, the last three quantities $\dot{A}_{l_{1}}^{m \mu}\left(l_{1} l_{2} m r \mid l s\right)\left\{l_{1} l_{2} v_{1} v_{2} \mid l v\right\}$ being purely mathematical quantities.

Equation (30) establishes a relationship between the starting $\gamma$-texture, the crystallographic transformation law, the variant selection function and the resulting $\alpha$-texture. 
The point is to know whether any one of these quantities can be determined if the other three are known.

\section{CALCULATION OF THE A-TEXTURE}

The most straightforward problem is to calculate the $\alpha$-texture if the starting $\gamma$-texture is known. This is possible if the coefficients $W$ of the transformation function and $V$ of the variant selection function are known.

A difficulty appears, however, in eq. (30). In order to calculate the coefficients ${ }^{\alpha} C$ of degree $l$ the coefficients ${ }^{\gamma} C$ of all degrees $l_{1}$ from zero up to infinity are needed. The same holds for the coefficients $V$ and the degree $l_{2}$. This is a result of the occurrence of a product of functions $v$ and $f_{\gamma}$ of the same variable in eq. (12) which has to be expressed by the Clebsch-Gordan coefficients if the series expansion is to be used. Hence, we have first to truncate the series with respect to $l_{1}$ and $l_{2}$ arbitrarily at certain values $L_{1} L_{2}$, just as we truncate $l$ at the value $L$.

Furthermore, since the coefficients ${ }^{\alpha} C$ of degree $l$ depend on the coefficients ${ }^{\gamma} C$ of all degrees $l_{1}$, it is no longer possible to treat the even and odd parts of the respective texture functions (Matthies 1979, Bunge and Esling 1979) separately. Equation (30) can be written

$$
{ }^{\alpha} C_{l}^{\rho v}=\sum_{l_{1}=0}^{L_{1}} \sum_{\mu=1}^{M\left(l_{1}\right)} \sum_{v_{1}=1}^{N\left(l_{1}\right)}{ }^{\gamma} C_{l_{1}}^{\mu v_{1}} \cdot a_{l l_{1}}^{\rho \nu \mu v_{1}}, \quad l \leqslant L
$$

with the known coefficients

$a_{l l_{1}}^{\rho v \mu v_{1}}=\frac{1}{2 l+1} \sum_{s=-1,2=0}^{+l} \sum_{m=-l_{1_{-}} \nu_{2}=1}^{L_{2}} \sum_{l}^{+l_{1}} \sum_{l_{2}}^{N\left(l_{2}\right)} V_{l_{2}}^{r v_{2}} \dot{A}_{l_{1}}^{m \mu}\left(l_{1} l_{2} m r \mid l s\right)\left\{l_{1} l_{2} v_{1} v_{2} \mid l v\right\}$

If the coefficients ${ }^{\gamma} C$ in eq. (31) have been determined experimentally, the coefficients ${ }^{\alpha} C$ of the texture after transformation can be calculated.

\section{CALCULATION OF THE T-TEXTURE}

In many cases it is, however, more important to calculate the starting 
texture $f_{\gamma}$ when the resulting texture $f_{\alpha}$ has been measured. This problem is of special interest in the case of the $\gamma \rightarrow \alpha$ transformation of steels. The hot-rolling of steels is usually carried out in the $\gamma$-region thus giving rise to a rolling or recrystallization (or an intermediate) texture in the $\gamma$-region which then transforms into the texture in the $\alpha$-region. Whereas it is extremely difficult in many practical cases to measure the $\gamma$-texture directly, it is easy to determine the $\alpha$-texture (Pospiech et al. 1973).

In order to solve this problem, the coefficients $a$ in eq. (31) must be known. The problem is then solve eq. (31) for the unknown coefficients ${ }^{\gamma} \mathrm{C}$ when the coefficients ${ }^{\alpha} \mathrm{C}$ are known.

A unique solution will in general be obtainable when the number of unknowns is not larger than the number of equations. The numbers of coefficients ${ }^{\alpha} C$ as well as of coefficients ${ }^{\gamma} C$ depend on the degrees $L$ and $L_{1}$ and on the respective crystal and sample symmetries. It seems reasonable to set $L_{1}=L$. Furthermore, the sample symmetry must remain unchanged. This means that $v$ and $v_{1}$ take on the same number of values.

A solution can thus be obtained for eq. (31) if the number of possible values $\mu$ is not larger than the number of possible values of $\rho$ and this in turn requires that the symmetry of the $\alpha$-crystals is not higher than the symmetry of the $\gamma$-crystals

$$
\operatorname{sym} \alpha \leqslant \operatorname{sym} \gamma
$$

In the $\gamma \rightarrow \alpha$ transformation of steels both symmetries are cubic. $\left.{ }^{*}\right)$ Equation (33) is thus fulfilled in this case. If so, eq. (31) will, as a rule, be soluble with coefficients ${ }^{\gamma} \mathrm{C}$ as unknowns. Hence, if the coefficients $a$ are known, the starting $\gamma$-texture can be recalculated from the resulting $\alpha$-texture.

It should be noted that these theoretical model calculations of the initial $\gamma$-texture, starting from the final $\alpha$-texture, may be compared with experiment in the favorable case of some high-alloy steels, such as $\mathrm{Fe} 30 \mathrm{Ni}$, whose phases $\gamma$ and $\alpha$ are both stable at room temperature (Kallend et al. 1976, Davies et al. 1976, Bateman and Davies 1981, Wagner et al. 1981, Esling and Bechler 1982).

\footnotetext{
(*) martensite, strictly tetragonal, can be annealed at $250^{\circ} \mathrm{C}$ to give cubic martensite probably without changing texture.
} 


\section{CALCULATION OF THE TRANSFORMATION COEFFICIENTS A}

The transformation coefficients $a$ can be calculated if the coefficients $W$ and $V$ of the transformation function $w$ and the variant selection function $v$ are known. The point is then to know whether these coefficients can also be calculated from experimental data, i.e. corresponding values of ${ }^{\gamma} \mathrm{C}$ and ${ }^{\alpha} \mathrm{C}$. This would be possible only if as many pairs of textures were available as there are terms in the summation eq. (31), and all these different textures would have to be related with the same coefficients $a$. As we have already mentioned the variant selection function is assumed to depend on the metallurgical variables which must be varied in order to produce different textures. This shows that a direct experimental determination of the transformation coefficients seems not to be possible without additional assumptions. We shall see, however, that the calculation can be carried out if we make certain reasonable hypotheses concerning the orientation transformation function $w(\Delta g)$.

\section{The orientation transformation function $w$}

The orientation relation between the $\gamma$ and $\alpha$ crystals is often assumed to be a well-defined crystallographic relationship as in eq. (5). In many cases these are the Kurdjumov-Sachs (1930) or Nishiyama (1934)Wassermann (1933) relations which may be expressed by

$$
\begin{aligned}
& (111)_{\gamma} \|(110)_{\alpha}, \Delta g_{K S}=\left\{48^{\circ}, 85.7^{\circ}, 9.6^{\circ}\right\} \\
& {[110]_{\gamma} \|[111]_{\alpha}} \\
& (111)_{\gamma} \|(110)_{\alpha}, \Delta g_{N W}=\left\{45^{\circ}, 83.1^{\circ}, 6.9^{\circ}\right\} \\
& {[211]_{\gamma} \|[011]_{\alpha}}
\end{aligned}
$$

where $\Delta g$ has been expressed in terms of Euler angles.

As has already been mentioned, there may be a certain spread about these ideal orientation relationships which may be expressed in terms of a distribution function $w(\Delta g)$ which has its maximum value at $\Delta g_{0}$ (i.e. $\Delta g_{K S}$ or $\Delta g_{N W}$ of eq. (34), (35)). If the orientation relation is a strict one, with no spread about $\Delta g_{0}$ then the coefficients $W$ in eq. (32) take on the form 


$$
W_{l}^{\rho s}=(2 l+1) \dot{\vdots}_{l}^{\rho s}\left(\Delta g_{0}\right)
$$

where the left-side symmetry of the function $T$ is the crystal symmetry of the $\alpha$-crystals.

With a more realistic assumption, we may assume a spread about $\Delta g_{0}$ in the form of a Gauss distribution which drops to $e^{-1}$ at the angle $\omega_{0}$. Equation (36) is then to be replaced by

$$
W_{l}^{\rho s}=T_{l}^{\rho s}\left(\Delta g_{0}\right) \cdot \frac{\exp \left(-\frac{1}{4} l^{2} \omega_{0}^{2}\right)-\exp \left(-\frac{1}{4}(l+1)^{2} \omega_{0}^{2}\right)}{1-\exp \left(-\frac{1}{4} \omega_{0}^{2}\right)}
$$

There are also examples in which several, crystallographically different orientation relationships occur, possibly with different frequencies (Kurdjumov and Sachs 1930, Esling et al. 1978). In this case the coefficients are the weighted mean values of those of the individual relationships

$$
W_{l}^{\rho s}=\sum_{i=1}^{I} W_{l}^{\rho s}(i) \cdot M(i)
$$

where $M(i)$ is the volume fraction of the $i$ th component.

In any case it may be assumed that the orientation transformation function is a rather sharp function, the series expansion of which does not converge rapidly.

\section{The variant selection function $\boldsymbol{V}$}

The variant selection function $v$ was assumed to depend on the orientation $g_{\gamma}$ of the $\gamma$-crystals. It was the purpose of this function to distinguish between different crystallographically equivalent orientations. The function was thus chosen without crystal symmetry. It must however be assumed that $v$ obeys the sample symmetry which should not be broken by a non-directional processes such as thermal treatment.

Several possible reasons for variant selection have been discussed (Wagner et al. 1981, Bateman and Davies 1981). It is common to all of them that they lead to a rather smooth angular dependence of $v(g)$ as compared with sharp angular dependence of the orientation relation function $w(\Delta g)$. The series expansion of $v(g)$ must thus be assumed to converge rapidly, i.e. there will be only a few coefficients $V$ different from zero. 
Further, whereas the orientation relation function $w(\Delta g)$ is assumed to be relatively insensitive to the metallurgical variables, the variant selection function will be strongly sensitive to those variables. It will be, therefore, a good approximation to set the coefficients $W_{l}^{\rho s}$ in eq. (32) as constant and to approximate them by one of the theoretical expressions eq. (36)-(38) with a reasonable choice of the ideal rotations $\Delta g_{0}$ and the corresponding spreads $\omega_{0}$. This assumption leaves only the coefficients $V$ to be determined from experiment. They can be obtained from eq. (30) if the coefficients ${ }^{\alpha} C$ and ${ }^{\gamma} C$ are known. In this case eq. (30) can be rewritten in the form

$$
{ }^{\alpha} C_{l}^{\rho v}=\sum_{l_{2}=0}^{L} \sum_{r=-l_{2}}^{+l_{2}} \sum_{v_{2}=1}^{N\left(l_{2}\right)} V_{l_{2}}^{r v_{2}} \cdot b_{l l_{2}}^{\rho v r v_{2}}
$$

with

$$
b_{l l_{2}}^{\rho v r v_{2}}=\sum_{l_{1}=0}^{L_{1}} \sum_{\mu=1}^{M\left(l_{1}\right)} \sum_{v_{1}=-l_{1}}^{+l_{1}} C_{l_{1}}^{\mu v_{1}} \cdot \alpha_{l l_{2} l_{1}}^{\rho v r v_{2} \mu v_{1}}
$$

and

$$
\alpha_{l l_{2} l_{1}}^{\rho v v_{2} \mu v_{1}}=\sum_{s=-l}^{+l} W_{l}^{\rho s} \dot{A}_{l_{1}}^{m \mu}\left(l_{1} l_{2} m r \mid l s\right)\left\{l_{1} l_{2} v_{1} v_{2} \mid l v\right\}
$$

where

$$
m=s-r
$$

As has been mentioned, the variant selection function is assumed to be a smooth function. This means that the number of unknowns $V$ in eq. (39) is small compared to the number of equations, which is related to the degree $L$ of the series expansion of the $\alpha$-texture. It will thus, as a rule, be possible to solve eq. (39) for the coefficients $V$, i.e. to determine the variant selection function when the coefficients $b$ are known. These coefficients, in turn, depend on the coefficients of the corresponding $\gamma$-texture which are assumed to be known experimentally. The coefficients $\alpha$ in eq. (40) are assumed to be known theoretically according to eq. (41). The latter equation contains the coefficients $W$ which we have assumed according to one of the transformation laws eq. (36)-(38). The other quantities in eq. (41) are purely mathematical quantities. 
Under these circumstances, it will thus be possible to determine the variant selection function if two corresponding textures $f_{\gamma}(g)$ and $f_{\alpha}(g)$ have been measured. As has been mentioned above, this has to be done for a variety of metallurgical parameters which may influence the variant selection.

Numerical calculations based on the above theory are presently being carried out and will be published in due course.

\section{SUMMARY}

Solid state phase transformations often occur in a crystallographically related way. During martensitic tranformation of steels, for example, the Kurdjumov-Sachs or Nishiyama-Wassermann relationships have been observed. Any preferred orientation of the crystallites in the starting phase will then be inherited, in some manner, by the newly formed crystals. This is called texture transformation. The problem of texture transformation has been treated mathematically in terms of the orientation distribution function and the orientation transformation function. In this analysis it has so far been assumed that crystallographically equivalent variants of the transformation law occur with equal probability. Experimental investigations have shown, however, that this is, in general, not the case. In this article a generalized treatment of texture transformation is presented, taking a variant selection function into account.

\section{References}

Bateman, R. M. and Davies, G. J. in The Sixth International Conference on Textures of Materials. The Iron and Steel Institute of Japan, Tokyo (1981).

Bunge, H. J. Mathematische Methoden der Texturanalyse. Akademie Verlag, Berlin (1969).

Bunge, H. J. and Esling, C. J. Physique Lett. 40, L-627 (1979).

Bunge, H. J. and Esling, C. C.R. Hebd. Séan. Acad. Sci. 289B, 163 (1979).

Bunge, H. J. and Esling, C. Quantitative Texture Analysis. Deutsche Gesellschaft für Metallkunde, Oberursel (1982).

Bunge, H. J. Texture Analysis in Materials Science-Mathematical Methods. Butterworth, London (1982).

Davies, G. J., Kallend, J. S. and Morris, P. P. Acta Met. 24, 159 (1976).

Esling, C. and Bechler, E. in Qualitative Texture Analysis. Deutsche Gesellschaft für Metallkunde, Oberursel (1982).

Esling, C., Wagner, F., Baro, R. and Englander, M., in Textures of Materials. SpringerVerlag, Berlin (1978).

Kallend, J. S., Morris, P. P. and Davies, G. J. Acta Met. 24, 361 (1976). 
Kurdjumov, G. and Sachs, G. Z. Phys. 64, 325 (1930).

Matthies, S. Phys. Status Solidi (b) 92, K135 (1979).

Nishiyama, Z. Sci. Rep. Tohuku Imp. Univ. I 23, 637 (1934).

Pospiech, J., Jura, J. and Macjosowski, A., in 3ème Colloque européen sur les textures. Pont-à-Mousson. (1973).

Sargent, C. M. Scripta Met. 8, 821 (1974).

Wagner, F., Bergmann, H. W., Humbert, M. and Esling, C., in The Sixth International Conference on Textures of Materials. The Iron and Steel Institute of Japan, Tokyo (1981).

Wassermann, G. Archiv. für das Eisenhütt. 16, 647 (1933). 